\title{
Analysis of genetic events that modulate the oncogenic and growth suppressive activities of the PAX3-FKHR fusion oncoprotein
}

\author{
Shujuan J Xia ${ }^{1}$, Prerna Rajput ${ }^{1,2}$, Donna M Strzelecki ${ }^{1}$ and Frederic G Barr ${ }^{1}$
}

\begin{abstract}
Alveolar rhabdomyosarcoma (ARMS) is associated with chromosomal translocations that generate PAX3-FKHR and PAX7FKHR fusion oncoproteins. Based on studies demonstrating that high PAX3-FKHR expression causes growth suppression, the hypothesis is proposed that, during ARMS tumorigenesis, the translocations cause low oncoprotein expression and are followed by collaborating events that block growth suppression pathways and permit upregulation of oncoprotein expression. To investigate oncogenic function at low expression levels, PAX3-FKHR was introduced into NIH3T3 cells in the pBabe retroviral vector. Compared to high expression systems, PAX3-FKHR expression from pBabe was lower and did not suppress growth, but showed transforming activity in the soft agar assay. As a possible collaborating event, PAX3FKHR paired box mutations were previously shown in high expression systems to reverse growth suppressive effects. In the low expression system, the paired box mutation enhanced transformation in soft agar and focus formation assays. Although these mutations are candidate collaborating events, sequencing of paired box regions in ARMS tumors did not identify mutations. Finally, genes from known genetic alterations in ARMS were introduced, alone or combined, into NIH3T3 cells with high PAX3-FKHR expression and did not rescue growth suppression. In summary, these studies provide a model for an event in ARMS tumorigenesis that enhances PAX3-FKHR oncogenicity and abrogates growth suppression, but do not demonstrate a known event occurring in ARMS tumors that fulfills these criteria.
\end{abstract}

Laboratory Investigation (2007) 87, 318-325. doi:10.1038/labinvest.3700521; published online 12 February 2007

KEYWORDS: chromosomal translocation; gene fusion; growth suppression; transformation; alveolar rhabdomyosarcoma

Alveolar rhabdomyosarcoma (ARMS) is an aggressive softtissue tumor associated with the skeletal muscle lineage that occurs mainly in children. ${ }^{1}$ Cytogenetic analyses revealed that recurrent chromosomal translocations, usually $\mathrm{t}(2 ; 13)$ (q35;q14) and less frequently $\mathrm{t}(1 ; 13)(\mathrm{p} 36 ; \mathrm{q} 14)$, occur in ARMS. These translocations generate chimeric genes that encode fusion proteins with the $\mathrm{N}$-terminal portion of PAX3 or PAX7 fused in-frame to the C-terminal region of FKHR. ${ }^{2}$ These fusion products are more highly expressed in ARMS cells, ${ }^{3}$ are more potent transcription factors, ${ }^{4}$ and are expressed exclusively in the nucleus ${ }^{5}$ compared to the corresponding wild-type (WT) products. Based on these abnormal features, these fusion proteins act as aberrant transcription factors that alter the pattern of downstream gene expression within the cell and contribute to tumorigenesis, as evidenced by transforming activity in the NIH3T3 soft agar assay. ${ }^{6-8}$
To further study the phenotypic consequences of fusion protein expression, PAX3-FKHR was introduced into NIH3T3 and other murine cells. ${ }^{8}$ These experiments were conducted using the retroviral vector $\mathrm{pK} 1$, which contains a strong promoter, expressing the test and puromycin resistance genes as a single transcript with an internal ribosome entry site to permit translation of two proteins. In contrast to control cells that progressively grew, cells expressing PAX3FKHR diminished in number over a 6-day period at which point a population of cells started to grow. Western blot analysis showed that the initial population expressed high PAX3-FKHR protein levels, comparable to the level in ARMS cells, whereas the surviving population expressed $\sim 10$-fold lower levels. These findings indicate that high PAX3-FKHR levels exert growth suppressive effects and are not tolerated by the murine cells whereas ARMS cells tolerate these high

\footnotetext{
'Department of Pathology and Laboratory Medicine, University of Pennsylvania School of Medicine, Philadelphia, PA, USA and ${ }^{2}$ School of Surgery and Pathology, University of Western Australia, Nedlands, WA, Australia

Correspondence: Dr FG Barr, MD, PhD, Department of Pathology and Laboratory Medicine, University of Pennsylvania School of Medicine, 505C Stellar Chance Laboratories, 422 Curie Boulevard, Philadelphia, PA 19104, USA. E-mail: barrfg@mail.med.upenn.edu

Received 16 October 2006; accepted 21 November 2006
} 
levels and thus have mechanisms for attenuating the suppressive effects.

Based on these findings, we postulate a scenario for ARMS tumorigenesis. In this scenario, we propose that an early event is the 2;13 translocation, which generates low PAX3FKHR expression and induces transformation. We then propose that one or more additional genetic alterations occur to collaborate with PAX3-FKHR, and to abolish growth suppression pathways that would be triggered by later high PAX3-FKHR expression. Later in ARMS tumorigenesis, we propose that PAX3-FKHR upregulation occurs and would not induce growth suppression due to the collaborating events that attenuated these effects. The high PAX3-FKHR level would then be able to induce additional biological effects.

In this report, we conducted studies to test and advance this scenario. To model putative early events in ARMS tumorigenesis, we developed an inducible system that expresses lower levels of PAX3-FKHR and does not demonstrate growth suppression. In this system, we can examine the effect of putative collaborating events on oncogenic activity without interference from the growth suppressive activity. As potential collaborating events, we further analyzed the oncogenic effects of PAX3-FKHR-binding domain mutations, and then proceed to screen ARMS tumors for sequence alterations in these domains. Finally, to investigate how other genetic alterations previously identified in ARMS cells impact on growth suppression, we used the inducible pK1-based murine cell systems and assayed whether these alterations (singly or combined) affected the level of PAX3-FKHR-induced growth suppression.

\section{MATERIALS AND METHODS Retroviral Constructs}

The PAX3-FKHR-ER fragment was subcloned into the pBabe-puro retroviral vector, and G48S and N269A mutations were introduced using corresponding primers ${ }^{8}$ and the Quik-change site-directed mutagenesis kit (Stratagene). To generate the PAX3-FKHR-pBabe construct, a stop codon was introduced into the $3^{\prime}$ end of PAX3-FKHR in PAX3-FKHRER-pBabe. The SV40 large T antigen and CDK4 were subcloned into MigR1, a retroviral vector with a GFP marker (provided by Dr W Pear). MDM2 was subcloned into pR-Zeo, a retroviral vector constructed from MigR1 with a zeocin resistance marker, and MYCN was subcloned into $\mathrm{pK}-\mathrm{Hyg}$, a retroviral vector constructed from $\mathrm{pK} 1$ with a hygromycin resistance marker.

\section{Cell Culture, Transduction, and Cell Growth Assays}

NIH3T3 cells were cultured in DMEM medium (Gibco) supplied with $10 \%$ fetal bovine serum (Hyclone) and antibiotics-antifungizone (Gibco) at $37^{\circ} \mathrm{C}, 5 \% \mathrm{CO}_{2}$ and $95 \%$ humidity. The retroviral transduction procedure was performed as described previously. ${ }^{8}$ Antibiotic selection was performed as follows: pK1 and pBabe-transduced cells were selected in $3 \mu \mathrm{g} / \mathrm{ml}$ puromycin (Clontech), pR-Zeo-transduced cells in $1 \mathrm{mg} / \mathrm{ml}$ zeocin (Invitrogen), and pK-Hygtransduced cells in $600 \mu \mathrm{g} / \mathrm{ml}$ hygromycin (Roche). In addition, MigR1-transduced cells were harvested using fluorescent activated cell sorting analysis (FACS, Becton Dickinson). For cell growth analysis, $2 \times 10^{4}$ cells/well were seeded in a six-well plate (Corning), and cell number was counted by the trypan blue exclusion method (Gibco) at the indicated times with or without 4-hydroxytamoxifen (TMF) (Sigma). Cell growth is expressed relative to the cell number at day 1 .

\section{Western Blot Analysis and Luciferase Reporter Assays} Cells were lysed using RIPA buffer (Santa Cruz Biotechnology) and protein concentration was quantified with the Bio-Rad Protein Assay Kit. Forty micrograms of protein were fractionated in $4-12 \%$ Nupage gel (Invitrogen) and transferred onto nitrocellulose filter (Hybond-ECL, Amersham Biosciences). The antibodies used in this work were: anti-PAX3 rabbit polyclonal (Abcam), anti-CDK4 rabbit polyclonal (C-22, Santa Cruz Biotechnology), anti-MDM2 monoclonal (Ab-1, CalBiochem), anti-MYCN rabbit polyclonal (C-19, Santa Cruz Biotechnology), anti-SV40 large T antigen monoclonal (Pab-101, Santa Cruz Biotechnology) and anti-actin goat polyclonal (I-19, Santa Cruz Biotechnology).

For the luciferase assay, $2 \times 10^{4}$ cells were seeded in each well of a 24-well plate, and transfected using Fugene 6 reagent (Roche) with $0.3 \mu \mathrm{g}$ of firefly luciferase reporter $(6 \times$ PRS9, $6 \times \mathrm{CD} 19$ or $1 \times \mathrm{P} 3)$ and $0.015 \mu \mathrm{g}$ of renilla luciferase reporter (PRL-TK, control for transfection efficiency). After $24 \mathrm{~h}$, the cells were treated with or without $30 \mathrm{nM}$ TMF (Sigma) for a $24 \mathrm{~h}$ period, and then the dual luciferase assay was performed using the reporter assay kit (Promega).

\section{Transformation Assays}

For the soft agar colony assay, $2 \times 10^{4}$ cells in Iscove's medium (Gibco) containing 10\% FBS with $0.35 \%$ agar (Agar Noble, Difco Laboratories) were seeded on top of $0.7 \%$ agar in the same medium. Both agar layers also contain the specified concentration of TMF. Two milliliters of medium with $0.7 \%$ agar and the specified concentration of TMF were added once per week. After three weeks incubation, the colonies were counted.

For the focus formation assay, $1 \times 10^{3}$ transduced cells were mixed with $10^{6}$ untransduced NIH3T3 cells and seeded into a $10 \mathrm{~cm}$ dish with $15 \mathrm{ml}$ DMEM medium containing $10 \%$ FBS and the indicated TMF concentration. The medium with the indicated TMF concentration was changed two times per week for 2 weeks. After 2 weeks, the medium was removed, the cells were treated with Giemsa staining solution (Sigma), and the foci were counted. 


\section{Sequence Analysis of the DNA-Binding Domains of Fusion Positive ARMS Tumors}

The Primer3 program (http://frodo.wi.mit.edu/cgi-bin/ primer3/primer3_www.cgi) was used to select primer pairs that span the entire paired box or homeodomain of PAX3-FKHR and PAX7-FKHR (Table 1). Total RNA was extracted from frozen tumors and RT-PCR was performed, as described previously. ${ }^{9}$ The RT-PCR products were electrophoresed on a $1 \%$ agarose gel and the DNA was isolated using a QIAquick gel extraction kit (Qiagen). Using a series of primers on both strands of these regions, the paired box or homeodomain in the purified RT-PCR products was completely sequenced (50 ng of DNA and $1.1 \mu \mathrm{M}$ of primer) in the University of Pennsylvania DNA Sequencing Facility. The DNA sequences were aligned against a known PAX3-FKHR and PAX7-FKHR sequence using Sequencher (Gene Codes) to check for sequence alterations.

\section{RESULTS}

\section{Low Level PAX3-FKHR Expression does not Suppress Growth and Promotes Transformation}

Our previous data indicated that high PAX3-FKHR expression from pK1 suppresses NIH3T3 cell growth. ${ }^{8}$ By fusing PAX3-FKHR to the estrogen receptor (ER) ligand-binding domain, a TMF-inducible system was generated in $\mathrm{pK} 1$ and showed increased growth suppression and decreased transformation as PAX3-FKHR activity increased. These findings indicate the oncogenic consequences in a high level PAX3FKHR expression system without mechanisms for attenuating growth suppression. To examine our ARMS tumorigenesis scenario, we developed a cell culture system for studying oncogenic consequences at low PAX3-FKHR expression levels. In this model system, PAX3-FKHR was again linked in-frame with the ER ligand-binding domain so that transcription activity is manipulated by addition of TMF. This

\section{Table 1 PCR primers for DNA binding domain regions of PAX3-FKHR and PAX7-FKHR}

\begin{tabular}{ll}
\hline Paired box primers & Sequence $\left(5^{\prime}-3^{\prime}\right)$ \\
\hline P3FWPB & 5'-GT CCC GCT GGA AGT GTC-3' \\
P3RVPB & 5'-GCC TCC TTC CTC TCC AAG TC-3' \\
P7FWPB & 5'-ACG GGA TTC CCT TTG GAA G-3' \\
P7RVPB & 5'-GAT GCT GTG Tा GGC CT C-3' \\
& \\
Homeodomain primers & Sequence $\left(5^{\prime}-3^{\prime}\right)$ \\
\hline P3FWHD & \\
P3RVHD & 5'-AGG AAG GAG GCA GAG GAA AG-3' \\
P7FWHD & 5'-GGT AAG AGT GCT CCG ACA GC-3' \\
P7RVHD & 5'-AAG AAG GCC AAA CAC AGC AT-3' \\
& 5'-GTG GTG GGG TAG GTG GAG T-3' \\
\hline
\end{tabular}

construct was then subcloned into the pBabe retroviral vector, which differs from $\mathrm{pK} 1$ in that the test and puromycin resistance genes are expressed as separate transcripts, and thus high level PAX3-FKHR expression is not enforced by antibiotic selection. In addition, expression in pBabe is driven from a murine leukemia virus LTR in contrast to the murine stem cell PCMV virus LTR, which drives high level expression in MSCV-based vectors such as $\mathrm{pK} 1$ and MigR1. After transduction into NIH3T3 cells and selection in puromycin, Western blotting demonstrated that the expression level of PAX3-FKHR-ER in pBabe is much lower than that from $\mathrm{pK} 1$ (Figure 1a). In addition, a reporter assay using the luciferase gene linked to PAX3 DNA-binding sites revealed that reporter activity is much lower in cells transduced with PAX3-FKHR-ER-pBabe compared with PAX3-FKHRER-pK1 under the same TMF treatments (Figure 1b).

Next, we measured the growth of cells transduced with PAX3-FKHR-ER in pBabe and explored the effect of lower PAX3-FKHR expression on NIH3T3 cell growth. In NIH3T3 cells expressing PAX3-FKHR-ER in pK1, treatment with as little as $10 \mathrm{nM}$ TMF resulted in detectable growth suppression (Figure $4 \mathrm{~b}$ and Xia and $\mathrm{Barr}^{8}$ ). In contrast, PAX3-FKHR-ER expressed from pBabe vector did not inhibit NIH3T3 cell growth when treated with as high as $100 \mathrm{nM}$ TMF (Figure 1c). These data confirm that the expression level of PAX3FKHR is critical for NIH3T3 cell growth; higher level expression inhibits cell growth, but expression below a lower threshold does not detectably affect cell growth. Therefore, this pBabe expression system provides the opportunity to model our postulated first step in ARMS tumorigenesis and thereby to study PAX3-FKHR transformation without accompanying growth suppression associated with high PAX3-FKHR expression.

To examine the relationship between transformation and PAX3-FKHR activity in this low expression system, the PAX3-FKHR-ER-transduced NIH3T3 cells were seeded in soft agar and treated with varying TMF doses. The results show that PAX3-FKHR promotes soft agar colony formation in a TMF dose responsive manner using this pBabe system (Figure 1d). Therefore, these findings demonstrate that low PAX3-FKHR expression is a potent inducer of cellular transformation and suggests that, under an expression threshold, increasing activity/expression results in increased oncogenic output.

Next, to independently assess the phenotypic consequences of low PAX3-FKHR levels, this fusion was expressed constitutively in NIH3T3 cells using pBabe. The fusion protein was detected by Western blot analysis, and was active in the reporter assay (Figure 2a and data not shown). The expression level of PAX3-FKHR from pBabe is lower than that from pK1 when expressed transiently (after 3 days puromycin selection) (Figure 2a), but both vectors express similar PAX3FKHR levels after long-term selection. Cell growth assays demonstrated that these PAX3-FKHR expressing cells grew at a rate similar to control cells, and thus these cells did not 
a
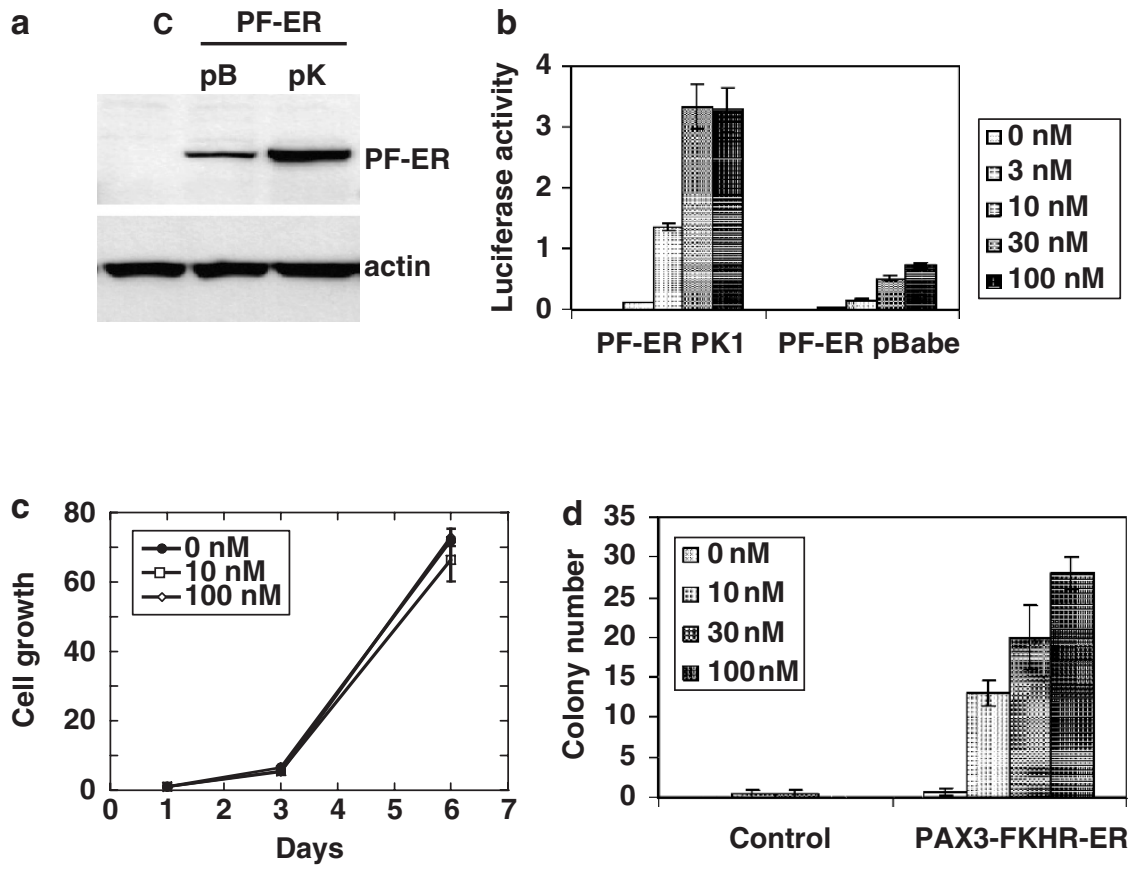

Figure 1 Effects of low level inducible PAX3-FKHR construct on transformation and growth suppression in NIH3T3 cells. (a) Western blot analysis of PAX3FKHR-ER protein expression in pBabe and pK1 retroviral constructs. Lanes (loaded with $40 \mu \mathrm{g}$ cell extract) containing vector control (C) and PAX3-

FKHR-ER (PF-ER) in pBabe $(\mathrm{pB})$ and $\mathrm{pK} 1$ transduced cells are labeled on the top, and positions of PAX3FKHR-ER and actin loading control are labeled the right. (b) Comparison of transcriptional activity of PAX3-FKHR-ER-transduced into NIH3T3 cells using pBabe and pK1 inducible systems. Dual luciferase assay was performed using PRS9 luciferase reporter with indicated concentrations of 4-hydroxytamoxifen (TMF). The luciferase activity was normalized for transfection efficiency and expressed as the mean ( \pm s.d.) of triplicate assays. (c) Cell growth of NIH3T3 cells expressing PAX3-FKHR-ER in pBabe. Triplicate cultures of PAX3-FKHR-ERtransduced cells were treated with the indicated concentration of TMF, and counted at the indicated time points. The cell numbers were normalized to the first day counts and expressed as the mean ( \pm s.d.) of triplicate assays. (d) Soft agar colony assay of PAX3-FKHR-ER-transduced NIN3T3 cells. Cells were incubated with the indicated TMF concentration in soft agar and scored after three weeks. Three plates were counted for each TMF dose and results expressed as the mean ( \pm s.d.). demonstrate evidence of PAX3-FKHR-induced growth suppression (Figure 2b). Finally, the soft agar assay showed that this low level of PAX3-FKHR expression promoted cellular transformation at a level comparable to the inducible system (Figure 2c).

\section{Effect of PAX3-FKHR-Binding Domain Mutations in Low Expression System}

To dissect the contributions of the paired box and homeodomain to the phenotypic activities of the PAX3-FKHR fusion protein, we previously introduced mutations into each domain: glycine was replaced with serine at position 48 in the paired box (G48S), and asparagine was replaced with alanine at position 269 in the homeodomain (N269A). Using high level expression retroviral systems (pK1 or MigR1), we previously demonstrated that the homeodomain mutation abrogates transformation but has no effect on growth suppression. In contrast, at high expression levels, the paired box mutation, partially abrogates growth suppression and may promote transformation. Therefore, the paired box mutation is a potential collaborating genetic alteration that may help to attenuate PAX3-FKHR-induced growth suppression. The question arises as to whether this mutation has any selective effects when PAX3-FKHR is expressed at low levels in accord with our proposed model of ARMS tumorigenesis. To assess the effects on transformation, WT and the two mutated versions of PAX3-FKHR-ER, all contained within pBabe, were transduced into NIH3T3 cells. Western blot analysis of the cell lysates showed that the expression levels are comparable in these transduced lines (Figure 3a). Dual luciferase reporter assays were performed using specific DNA-binding sites (CD19 for paired box and P3 for homeodomain) and verified the loss of function of the mutations introduced into the corresponding domains (Figure $3 \mathrm{~b}$ ).

Next, we explored the effect of these mutant forms of PAX3-FKHR on cell growth and transformation in NIH3T3 cells. In the cellular growth assay, similar to WT PAX3FKHR, even as the TMF concentration was increased to $100 \mathrm{nM}$, the two PAX3-FKHR mutants did not show any detectable growth suppression (data not shown). Therefore, we could evaluate the effects of these mutations on oncogenic transformation in the absence of any concomitant growth suppression. In the soft agar assay of anchorage independence, the N269A mutant failed to induce any significant soft agar colony formation at any TMF dose, confirming that the homeodomain is required for PAX3FKHR to induce transformation (Figure 3c). In contrast, as the TMF concentration was increased, the G48S mutant enhanced soft agar colony formation dramatically in a dose responsive manner. The extent of colony formation was substantially higher than in WT PAX3-FKHR expressing cells (Figure 3c), indicating a novel mechanism for increased stimulation of PAX3-FKHR induced transformation. To further explore the effect of the G48S mutation on oncogenic transformation, we employed the focus formation assay, which detects loss of cell contact inhibition. Analysis of focus formation in cells transduced with WT PAX3-FKHR as well as the vector alone or the $\mathrm{N} 269 \mathrm{~A}$ mutant did not reveal any 

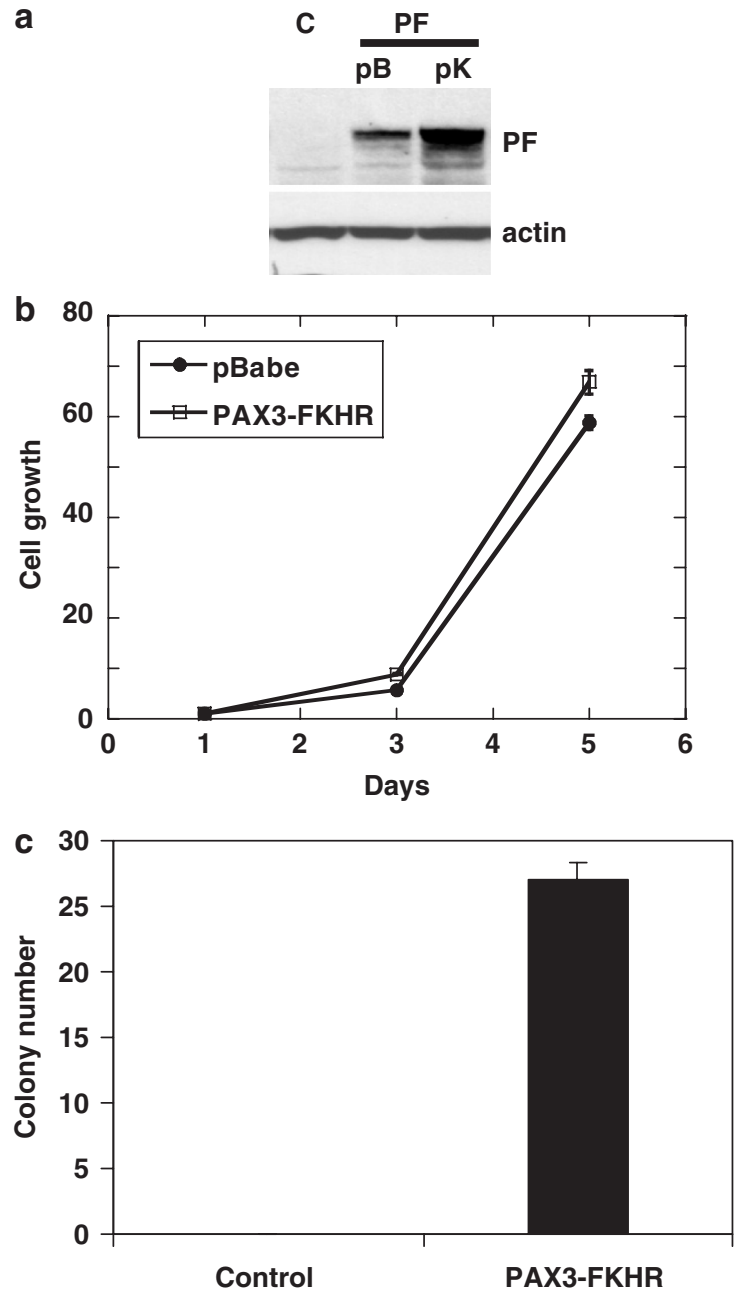

Figure 2 Effects of low level constitutive PAX3-FKHR expression on transformation and growth suppression in NIH3T3 cells. (a) Western blot analysis of PAX3-FKHR expression in pBabe- and pK1-transduced cells. Lanes (loaded with $40 \mu \mathrm{g}$ cell extract) containing vector control (C) and PAX3-FKHR in pBabe and PAX3-FKHR in pK1-transduced cells are labeled on the top, and positions of PAX3-FKHR (PF) and actin loading control are labeled at the right. (b) Cell growth of NIH3T3 cells transduced with PAX3FKHR in pBabe. Triplicate cultures of PAX3-FKHR or vector-transduced cells were counted at the indicated time points. The cell numbers were normalized to the first day counts and expressed as the mean $( \pm$ s.d.) of triplicate assays. (c) Soft agar colony assay of NIH3T3 transduced with PAX3-FKHR in pBabe. PAX3-FKHR or vector-transduced cells were plated in soft agar and scored after three weeks. Three plates were counted for each construct and results were expressed as the mean ( \pm s.d.).

significant focus formation on a monolayer of NIH3T3 cells. However, the G48S mutant promotes clearly detectable focus formation in response to TMF (Figure 3d). These findings indicate that an inactivating mutation in the paired box increases the oncogenic potency of PAX3-FKHR and thus has selective value in the setting of low PAX3-FKHR expression. In combination with the finding that this mutation attenuates growth suppression at high PAX3-FKHR expression levels, such an alteration is a model of and candidate for a collaborating event in our model of ARMS tumorigenesis.

\section{Screening for PAX3-FKHR and PAX7-FKHR-Binding Domain Mutations in ARMS Tumors}

As an inactivating mutation of the paired box in PAX3-FKHR conforms to the criteria for a collaborating event in our proposed scenario for ARMS tumorigenesis, we proceeded to determine whether such mutations actually occur in ARMS cells. To conduct a larger screen for fusion protein-binding domain mutations in fusion-positive ARMS tumors, we examined the regions encoding both the paired box and the homeodomain in tumors expressing either the PAX3-FKHR or PAX7-FKHR fusion transcript. RT-PCR was performed to isolate each of these regions from tumor RNA as single DNA fragments. As these fusion genes are overexpressed in tumor cells relative to the corresponding WT gene, ${ }^{3}$ the use of RNA as a starting material will enrich for binding domains originating from the fusion gene. The DNA sequencing results revealed that there were no significant mutations present within the paired box (23 samples) and homeodomain (19 samples) of PAX3-FKHR ARMS tumors or in the paired box (11 samples) and homeodomain (10 samples) of PAX7FKHR ARMS tumors. Therefore, although the paired box mutations provided a collaborating event in the cell culture model system, these mutations are not in vivo events in ARMS tumors.

\section{Search for Collaborating Events that Attenuate PAX3-FKHR-Induced Growth Suppression}

As an alternative approach for identifying collaborating events consistent with our ARMS tumorigenesis scenario, we turned to in vivo events identified in ARMS tumors and assayed if they attenuate PAX3-FKHR-induced growth suppression. Comparative genomic hybridization analyses of ARMS tumors have identified the two most frequently amplified regions in ARMS to be $2 \mathrm{p} 24$ and $12 \mathrm{q} 13-15 .{ }^{10,11}$ In the 2 p24 region, MYCN is amplified in a subset of ARMS cases. ${ }^{12}$ Although the region has not been extensively evaluated in ARMS, 12q13-15 amplicons in other sarcomas are centered around CDK4 and/or MDM2. ${ }^{13}$ To test whether overexpression of these genes affects PAX3-FKHR growth suppressive activity, MDM2, CDK4 and MYCN, alone or combined, were transduced into NIH3T3 cells expressing high level PAX3-FKHR-ER from the pK1 vector. After selection or FACS analysis, protein expression was confirmed by Western blotting analysis (Figure $4 \mathrm{a}$ ). Of note, expression of each protein remained relatively constant in the different combinations, and expression of these transduced genes did not interfere with expression of PAX3-FKHR-ER.

Next, we performed the cell growth assay with the inducible PAX3-FKHR-ER system to test if these co-expressed proteins attenuate growth suppression when PAX3-FKHR is activated by TMF. Our results show that CDK4, MDM2 or MYCN is not sufficient acting alone to overcome growth suppression in the presence of high PAX3-FKHR expression/ activity (Figure $4 \mathrm{~b}$ and data not shown). Furthermore, although combination of two or three oncoproteins promotes 

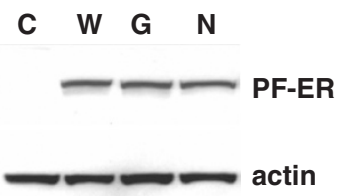

C

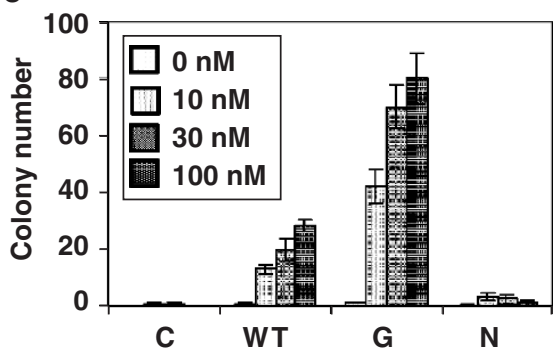

b

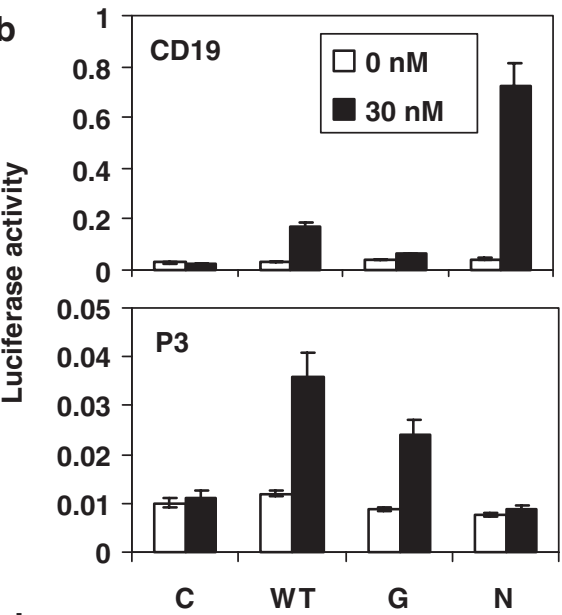

d

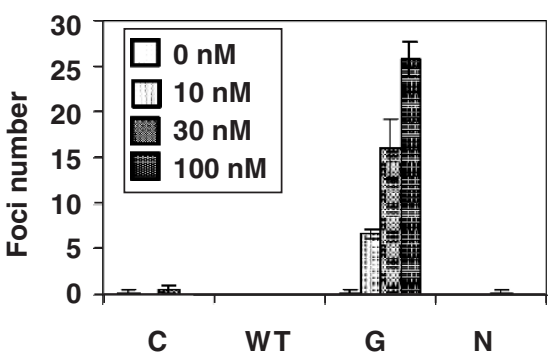

Figure 3 Effects of low expression of PAX3-FKHR paired box and homeodomain mutations on transformation in NIH3T3 cells. (a) Western blot analysis of mutant PAX3-FKHR-ER constructs in pBabe. Lanes (loaded with $40 \mu \mathrm{g}$ cell extract) containing vector control (C), wild-type (W), G48S (G) and N269A (N) mutated PAX3-FKHR-ER-transduced cells are labeled on the top, and positions of PAX3FKHR-ER (PF-ER) and actin loading control are labeled at the right. (b) Transcriptional activity of WT and mutated PAX3-FKHR-ER constructs in pBabe. The dual luciferase assay was performed using a luciferase reporter containing a CD19 (paired boxspecific) or a P3 (homeodomain-specific) binding site. This reporter was transfected in NIH3T3 cells transduced with vector control (C) or wild-type (WT), G48S (G) and N269A (N) PAX3-FKHR-ER constructs in pBabe, and treated with the indicated TMF concentrations. The luciferase activity was normalized for transfection efficiency and expressed as the mean ( \pm s.d.) of triplicate assays. (c) Soft agar colony assay of WT and mutated PAX3-FKHR-ER constructs in pBabe. NIH3T3 cells transduced with the corresponding constructs were incubated with the indicated TMF concentration in soft agar and scored after three weeks. Three plates were counted for each TMF dose and results expressed as the mean ( \pm s.d.). (d) Focus formation assay of WT and mutated PAX3-FKHR-ER constructs in pBabe. NIH3T3 cells transduced with the corresponding constructs were seeded with excess untransduced $\mathrm{NIH} 3 \mathrm{~T} 3$ cells in $100 \mathrm{~mm}$ dishes, and treated with the indicated concentrations of TMF. After 2 weeks, three plates were stained and counted for each TMF dose and results expressed as the mean ( \pm s.d.). cell growth compared to expression of PAX3-FKHR-ER alone (Figure $4 \mathrm{~b}$ ), various combinations of the three oncoproteins including a combination of all three in the same cell does not result in any significant change in growth suppression.

As MDM2 and CDK4 target the pRb and p53 pathways, we used the alternative approach of introducing the SV40 large $\mathrm{T}$ antigen as a single agent that will inactivate both $\mathrm{pRb}$ and $\mathrm{p} 53$ at the same time. After verifying the expression of T antigen by Western blotting analysis (Figure 4a), we determined that the SV40 large T antigen failed to rescue the growth suppression caused by PAX3-FKHR activation (Figure 4b). Therefore, disruption of $\mathrm{pRb}$ and/or p53 pathways does not enable cells to overcome PAX3-FKHR induced growth suppression.

\section{Transformed NIH3T3 Cells do not Attenuate Growth Suppression Induced by PAX3-FKHR}

In our previous report, ${ }^{8}$ PAX3-FKHR-ER in pK1 only efficiently transformed NIH3T3 cells in the soft agar assay at low TMF concentrations $(0-3 \mathrm{nM})$, and the transformation frequency decreased substantially as the TMF concentration was increased. As a final issue, we addressed the question whether the decrease in transformation frequency may be due to growth suppression of transformed cells induced by high levels of the oncoprotein. To investigate this issue, transformed colonies from these cells transduced with PAX3FKHR-ER in the pK1 vector were picked from soft agar plates in the presence of low TMF concentrations and expanded in culture as individual subclones. Western blot analysis of these subclones demonstrated varying levels of PAX3-FKHR-ER expression (data not shown). Two clones that expressed a PAX3-FKHR-ER level similar to the level in the original population were selected. When PAX3-FKHR activity was induced by $100 \mathrm{nM}$ TMF, the growth rate was suppressed in both clones, similar to the growth suppression seen in the parental population and demonstrating that transforming status does not give cells tolerance to PAX3-FKHR-induced growth suppression (Figure 5). Therefore, these findings support the premise that high PAX3-FKHR levels may transform cells but the associated growth suppression prohibits these transformed cells from proliferating to form detectable soft agar colonies.

\section{DISCUSSION}

In this study, we analyzed the relationship between PAX3FKHR fusion protein function and expression level, the effect of genetic alterations on fusion protein function, and applicability of these findings to actual alterations occurring in 

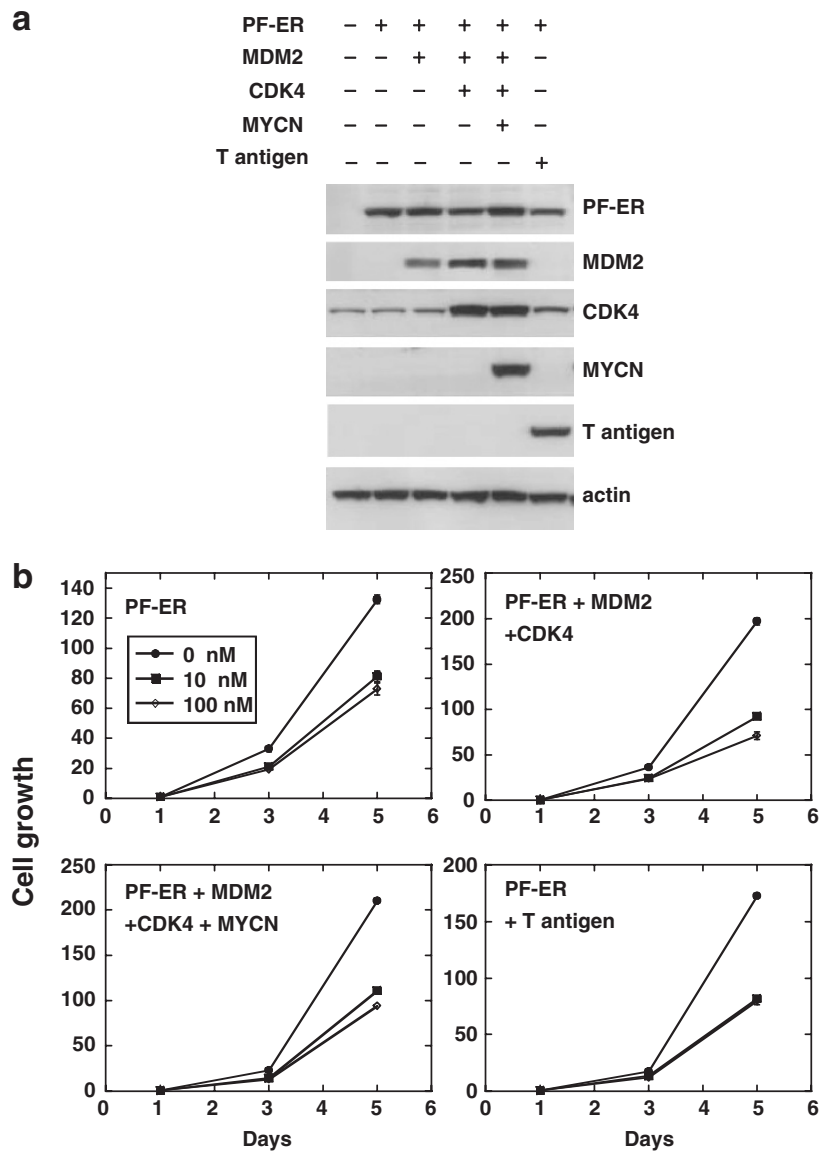

Figure 4 Effect of overexpression of MDM2, CDK4, MYCN and SV40 T antigen on growth of NIH3T3 cells expressing high level inducible PAX3FKHR construct. (a) Western blot analysis of PAX3-FKHR-ER (in pK1 vector), MDM2, CDK4, MYCN and SV40 T antigen. PAX3-FKHR-ER (PF-ER) and oncoproteins expressed in cells ( $40 \mu \mathrm{g}$ cell extract/lane) are indicated on the top, and positions of corresponding proteins, including actin loading control are labeled at the right. (b) Growth of $\mathrm{NIH} 3 \mathrm{~T} 3$ transduced with high level PAX3-FKHR-ER construct and additional oncogenes. Triplicate cultures of each set of transduced cells were treated with the indicated concentration, and counted at the indicated time points. The cell numbers were normalized to the first day numbers and expressed as the mean $( \pm$ s.d.) of triplicate assays.

ARMS tumors. Based on experiments described in this paper, an expression system is now available to evaluate the PAX3FKHR function at the low end of the expression range. As a result of differences between $\mathrm{pK} 1$ and pBabe retroviral vectors, there is a multi-fold difference in PAX3-FKHR expression such that high level expression from $\mathrm{pK} 1$ is associated with growth suppression and variable transforming activity whereas low level expression from pBabe is associated with transforming activity without growth suppression. Studies with transformed subclones revealed that the lower number of transformed cells seen at the higher expression levels is not due to lower intrinsic oncogenicity but rather to suppressed growth of transformed cells. Therefore, we conclude that oncogenic activity starts at very low expression levels and as expression increases, oncogenic activity increases but is
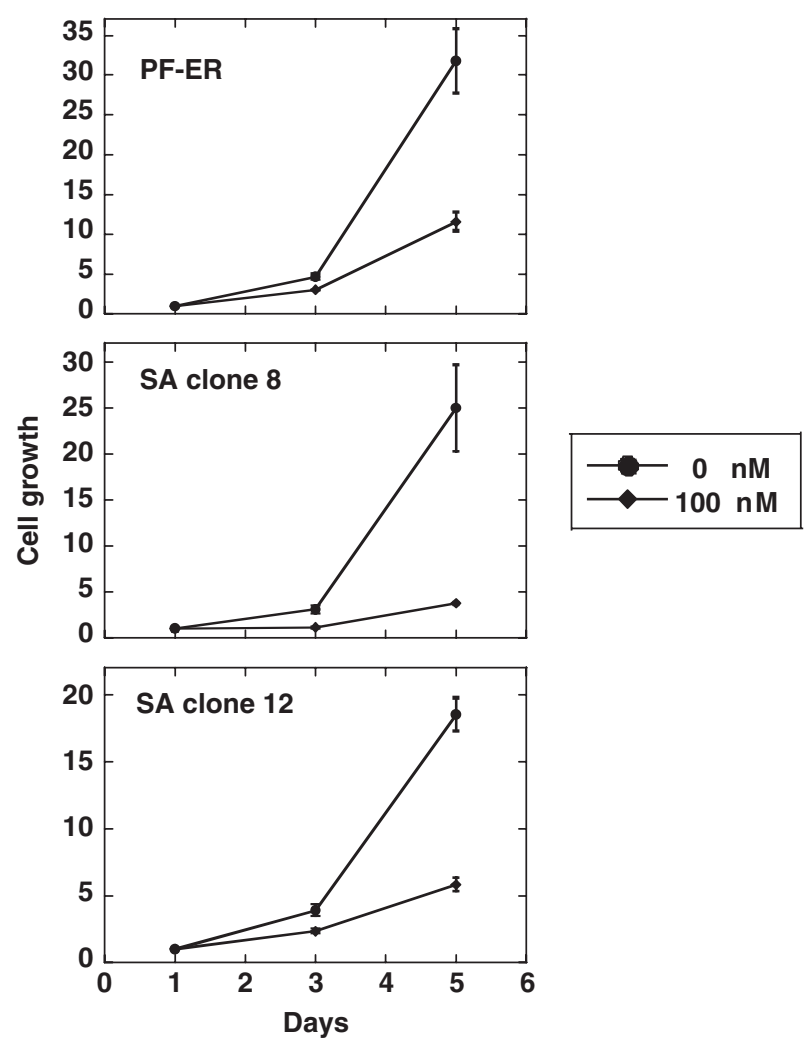

Figure 5 Growth status of NIH3T3 cells transformed by PAX3-FKHR. The original PAX3-FKHR-ER (from pK1) transduced population and cells from two transformed subclones (clones 8 and 12) derived from soft agar colonies were seeded in six-well plates. Triplicate cultures of each set of cells were treated with the indicated TMF concentration, and counted using trypan blue exclusion at the indicated time points. The cell numbers were normalized to the first day counts and expressed as the mean ( \pm s.d.) of triplicate assays.

antagonized and reversed by growth suppression, which commences at higher expression levels. The differing doseresponse curves for oncogenic and growth suppressive activities suggest that the target genes mediating these two different functions may be distinct. This hypothesis of separate targets is supported by the previous finding that growth suppression and transforming activities can be separately suppressed by paired box and homeodomain mutations, respectively. ${ }^{8}$ Alternatively, growth suppression may be the consequence of overexpressing oncogenic pathways, and thus some target genes may be the same with the target gene expression level being the key difference.

In the proposed scenario for ARMS tumorigenesis, separate low and high fusion gene expression steps are proposed with an intervening step of collaborating genetic alterations. In theory, such collaborating alterations could occur early in tumorigenesis and allow for immediate high level fusion gene expression without the need for an early low expression step. However, ARMS tumors are notable for fusion gene overexpression relative to the WT gene. For most tumors with the $1 ; 13$ translocation, the mechanism of overexpression is fusion 
gene amplification. ${ }^{3}$ Although a few tumors with the $2 ; 13$ translocation show fusion gene amplification, most cases appear to involve a copy-number independent increase in fusion gene transcription. We postulate that a transcriptional regulator is activated during tumorigenesis and preferentially upregulates the fusion gene. In favor of this hypothesis, previous studies identified some PAX3-FKHR ARMS cell lines in which PAX3-FKHR and PAX3 were expressed at comparable low levels. This finding suggests that either the transcriptional increase did not happen or was reversed, and is consistent with the hypothesis that low fusion gene expression is a distinct step in ARMS tumorigenesis.

The use of site-directed mutagenesis revealed a novel functional significance for the PAX3-FKHR paired box. At low expression levels, mutation of the paired box results in a striking increase in oncogenicity. At high expression levels, this mutation causes decreased growth suppression as well increased oncogenicity. In contrast, a homeodomain mutation inactivates oncogenic behavior. These findings confirm that PAX3-FKHR does not require two functional domains for oncogenic function. Moreover, these results contrast with studies of WT PAX3 in which critical mutations in either domain are associated with Waardenburg syndrome. ${ }^{14}$ Additional evidence indicates that the two domains are functionally interdependent in PAX3, and that the paired box regulates homeodomain specificity and dimerization. ${ }^{15}$ Based on this information, several hypotheses can explain the oncogenic consequences of the paired box mutation in PAX3FKHR. First, the paired box may function as an independent DNA-binding element and regulate a set of target genes that inhibit transformation, with mutation of the paired box removing such inhibitory effects and increasing transformation. Alternatively, since the two domains interact, inactivation of the paired box may increase homeodomainbinding specificity and dimerization potential, therefore enhancing expression of homeodomain-related target genes important for transformation.

Although one cannot rule out the possibility that there is some cell type uniquely tolerant of this fusion protein, the leading hypothesis to explain the growth suppressive effects of PAX3-FKHR in most cell types is that additional genetic alterations render ARMS cells tolerant to this protein. Although ARMS tumors often have amplification in addition to the translocation, ${ }^{11}$ several amplified targets (CDK4, MDM2, MYCN) did not alter PAX3-FKHR-mediated growth suppression, singly or in combinations. As CDK4, MDM2, and T antigen interfere with the pRB and p53 signaling pathways, these tumor suppressor pathways do not appear to be the source of PAX3-FKHR-mediated growth suppression. In contrast, a paired box mutation in PAX3-FKHR impairs growth suppression and stimulates oncogenicity and thus serves as a model for the collaborating mutation. The lack of paired box mutations in ARMS tumors indicates that such mutations are not the collaborating events occurring during in vivo ARMS tumorigenesis. Therefore, a novel mechanism must exist in the ARMS cell to collaborate with the fusion protein. One possibility is a non-mutagenic mechanism to inhibit paired box function, such as proteins binding to the paired box or post-translational modifications inhibiting paired box function. Additional studies are thus necessary to examine candidate alterations occurring in ARMS and further exploring novel mechanisms for inhibiting paired box function.

\section{ACKNOWLEDGEMENT}

We thank Ms Dara D Holder for technical assistance. This work was supported by NCl Grants CA64202, CA104896, and CA89461 (to FGB), and the Alveolar Rhabdomyosarcoma Research Fund

\section{DUALITY OF INTEREST}

The authors have no duality of interest to declare.

1. Xia SJ, Pressey JG, Barr FG. Molecular pathogenesis of rhabdomyosarcoma. Cancer Biol Ther 2002;1:97-104.

2. Barr FG. Gene fusions involving PAX and FOX family members in alveolar rhabdomyosarcoma. Oncogene 2001;20:5736-5746.

3. Davis RJ, Barr FG. Fusion genes resulting from alternative chromosomal translocations are overexpressed by gene-specific mechanisms in alveolar rhabdomyosarcoma. Proc Natl Acad Sci USA 1997;94:8047-8051.

4. Bennicelli JL, Edwards RH, Barr FG. Mechanism for transcriptional gain of function resulting from chromosomal translocation in alveolar rhabdomyosarcoma. Proc Natl Acad Sci USA 1996;93:5455-5459.

5. del Peso L, Gonzalez VM, Hernandez R, et al. Regulation of the forkhead transcription factor FKHR, but not the PAX3-FKHR fusion protein, by the serine/threonine kinase Akt. Oncogene 1999;18: 7328-7333.

6. Lam PY, Sublett JE, Hollenbach AD, et al. The oncogenic potential of the Pax3-FKHR fusion protein requires the Pax3 homeodomain recognition helix but not the Pax3 paired-box DNA binding domain. Mol Cell Biol 1999;19:594-601.

7. Du S, Lawrence EJ, Strzelecki D, et al. Co-expression of alternatively spliced forms of PAX3, PAX7, PAX3-FKHR and PAX7-FKHR with distinct DNA binding and transactivation properties in rhabdomyosarcoma. Int J Cancer 2005;115:85-92.

8. Xia SJ, Barr FG. Analysis of the transforming and growth suppressive activities of the PAX3-FKHR oncoprotein. Oncogene 2004;23: 6864-6871

9. Barr FG, Xiong QB, Kelly K. A consensus polymerase chain reactionoligonucleotide hybridization approach for the detection of chromosomal translocations in pediatric bone and soft tissue sarcomas. Am J Clin Pathol 1995;104:627-633.

10. Bridge JA, Liu J, Qualman SJ, et al. Genomic gains and losses are similar in genetic and histologic subsets of rhabdomyosarcoma, whereas amplification predominates in embryonal with anaplasia and alveolar subtypes. Genes Chromosomes Cancer 2002;33:310-321.

11. Gordon AT, Brinkschmidt C, Anderson J, et al. A novel and consistent amplicon at $13 q 31$ associated with alveolar rhabdomyosarcoma. Genes Chromosomes Cancer 2000;28:220-226.

12. Williamson D, Lu YJ, Gordon T, et al. Relationship between MYCN copy number and expression in rhabdomyosarcomas and correlation with adverse prognosis in the alveolar subtype. J Clin Oncol 2005;23: 880-888.

13. Berner JM, Forus A, Elkahloun A, et al. Separate amplified regions encompassing CDK4 and MDM2 in human sarcomas. Genes Chromosomes Cancer 1996;17:254-259.

14. Baldwin CT, Hoth CF, Macina RA, et al. Mutations in PAX3 that cause Waardenburg syndrome type I: ten new mutations and review of the literature. Am J Med Genet 1995;58:115-122.

15. Underhill DA, Gros P. The paired-domain regulates DNA binding by the homeodomain within the intact Pax-3 protein. J Biol Chem 1997;272:14175-14182. 